\title{
Realization of sneutrino self-interacting dark matter in the focus point supersymmetry
}

\author{
Bin Zhu, ${ }^{1, *}$ Ran Ding, ${ }^{2, \dagger}$ and Ying $\mathrm{Li}^{1, *}$ \\ ${ }^{1}$ Department of Physics, Yantai University, Yantai 264005, People's Republic of China \\ ${ }^{2}$ Center for High-Energy Physics, Peking University, Beijing, 100871, People's Republic of China
}

(Received 6 April 2018; published 6 August 2018)

\begin{abstract}
The lightest supersymmetric particle (LSP) is generally regarded as a Higgsino in focus point supersymmetry (SUSY). Under such a circumstance, it leads to a bileptino LSP when the minimal supersymmetric Standard Model (MSSM) is extended by the $U(1)_{B-L}$ gauge group within the framework of double focus point (DFP) supersymmetry. The bileptino is a copy of a Higgsino whose partner, the bilepton, is used to break $U(1)_{B-L}$ gauge symmetry spontaneously. Such a scenario, however, is not favored by direct detection since it leads to an unacceptable spin-independent cross section when one requires a correct self-scattering cross section. We point out that is not necessarily the case even in the presence of light $Z^{\prime}$. The right-handed sneutrino in this model acts as the LSP in most of the parameter space for nonvanishing soft trilinear coupling $T_{\eta}$. It is thus consistent with the requirement of DFP SUSY without involving any direct detection issue. The corrected relic abundance could be achieved via a sneutrino annihilating into a pair of bileptons, which also serve as a light mediator in self-interacting dark matter (SIDM). Moreover, the stringent constraint that comes from cosmic microwave background anisotropies can be evaded by considering the retarded decay of right-handed neutrinos. We further stress the need for a large soft trilinear term $T_{\eta}$ in order to generate a desirable self-scattering cross section $\sigma / m_{\tilde{\nu}_{R}}$ with moderate Yukawa coupling $Y_{\eta}$. The numerical calculation illustrates that SIDM is reliable in our model from the scales of a dwarf galaxy to a galaxy cluster.
\end{abstract}

DOI: 10.1103/PhysRevD.98.035007

\section{INTRODUCTION}

Supersymmetry (SUSY) [1] is a fermion-boson symmetry and is an indispensable layout of the Coleman-Mandula nogo theorem [2]. Its particle content is larger than the Standard Model (SM) in the sense that additional copied degrees of freedom are required, i.e., superpartners of SM particles. With the existence of superpartners at hand, especially the scalar top partner, the quadratic divergence in the loop correction of the Higgs mass is canceled even when SUSY is soft breaking [3], and the electroweak hierarchy problem is solved naturally. Our expectation of what to find beyond the SM has been deeply shaped by these naturalness arguments, and arguably low energy SUSY has emerged as the primary candidate for physics beyond Standard Model (BSM) physics. In addition, a discrete parity called $R$-parity is

\footnotetext{
*zhubin@mail.nankai.edu.cn

†dingran@mail.nankai.edu.cn

*liying@ytu.edu.cn
}

Published by the American Physical Society under the terms of the Creative Commons Attribution 4.0 International license. Further distribution of this work must maintain attribution to the author(s) and the published article's title, journal citation, and DOI. Funded by SCOAP ${ }^{3}$. imposed to forbid dangerous proton decay under which each particle bears a quantum number,

$$
P_{R}=(-1)^{3(B-L)+2 s},
$$

where $B, L$, and $s$ are, respectively, the baryon number, lepton number, and spin of the particle. This parity automatically guarantees that the lightest supersymmetric particle (LSP) is stable and could naturally qualify as a cold dark matter (CDM) candidate [4]. Therefore, the simplest realization of SUSY at the electroweak scale, minimal supersymmetric SM (MSSM), has led to the study of BSM physics during recent decades.

However, the situation is challenged by recent experiments. From the perspective of naturalness, the discovery of a $125 \mathrm{GeV}$ Higgs boson [5,6], together with nonobservation of sparticles in the LHC, put stringent limits on the parameter space of MSSM. This motivates us to consider other possible realizations of SUSY. There are in general two different approaches to improve the current issue: adding new fields or introducing a new SUSY breaking scenario. For the first approach, a well-known example is next-to-minimal supersymmetric Standard Model (NMSSM) [7-9], which improves fine-tuning through an additional tree-level F-term contribution on the Higgs mass. 
On the other hand, the focus point mechanism [10-20], which represents a second approach, keeps heavy enough sparticles to increase the Higgs mass while keeping the finetuning under control. The two schemes above can even be combined in a gauge extension SUSY framework, such as the $U(1)_{B-L}$ extended MSSM model (BLSSM) [21-25], which is the main focus of this paper.

On the dark matter side, the neutralino is severely limited by direct, indirect, and collider DM searches. Particularly, the vast parameter space of neutralino DM has been ruled out by direct detection experiments, even for the popular well-tempered neutralino [26]. In spite of these constraints, the CDM paradigm itself also encounters difficulty in interpreting small scale structure observations [27-29], which is known as the core-vs-cusp problem [30] and the too-big-to-fail problem [31]. Self-interacting dark matter (SIDM) [32-39] provides a valid solution to reconcile the above tension. Due to the above intriguing properties, SIDM has been extensively explored and the current status for this issue can be found, for instance, in Ref. [39]. In this scenario, the typical DM self-scattering cross section required for solving the small scale structure discrepancy is $\sigma / m_{\mathrm{DM}} \sim 1 \mathrm{~cm}^{2} / \mathrm{g}$ in galaxies, which is much larger than the weak-scale cross section preferred by the weakly interacting massive particle (WIMP) CDM model. That strongly invokes a light mediator to enhance the cross section via nonperturbative resummation.

Clearly, the neutralino fails to realize SIDM. To our knowledge, there is only one supersymmetric realization for SIDM in the literature, i.e., in the general next-tominimal supersymmetric SM (GNMSSM) [40] model. The underlying reason for choosing GNMSSM rather than scale invariant NMSSM is due to the fact that the vanishing of coupling $\lambda$ in the $\lambda S H_{u} H_{d}$ interaction (where $S$ denotes a singlet chiral superfield) is plausible, which makes the singlet sector decouple from the visible MSSM sector. Thus the $\{s, \tilde{s}\}$ sector provides a desirable singlino SIDM with an ultralight singlet Higgs being the force mediator. Inspired by such a strategy, we manage to obtain a similar sector of SIDM in the framework of BLSSM: the $U(1)_{B-L}$ extension of MSSM.

The phenomenology of BLSSM with heavy $Z^{\prime}$ [the gauge boson associated with $U(1)_{B-L}$ symmetry] has been extensively explored [21-23,41-43], and the case for a light $Z^{\prime}$ [44] is also interesting since it can account for a novel Be anomaly [45-48]. Notice that light $Z^{\prime}$ causes a similar small hierarchy problem as in the MSSM, which motivates the proposal of a double focus point (DFP) mechanism [24] to solve this problem. Within the framework of BLSSM with DFP, a viable choice for a SIDM candidate seems to be the bileptino with a light $Z^{\prime}$ in the $\left\{Z^{\prime}, \tilde{\eta}\right\}$ sector as a mediator. In this paper, however, we prove that this recipe will lead to a contradiction between the requirements of SIDM and limits from direct detection experiments for the bileptino LSP. This is mainly because the $Z^{\prime}$ could couple to quarks in terms of a nonvanishing gauge interaction; thus, the $\left\{Z^{\prime}, \tilde{\eta}\right\}$ sector does not hide itself from MSSM again. Alternatively, we propose the righthanded sneutrino as a SIDM candidate. The interesting point is that, in DFP, the sneutrino LSP requires a large trilinear soft term $T_{\eta}$, which is also the necessary condition of SIDM. We therefore obtain a natural SIDM with a $\left\{\tilde{\nu}_{1}, \eta\right\}$ sector, where the right-handed sneutino LSP $\tilde{\nu}_{1}$ and bileptons $\eta$ serve as the SIDM and force mediator, respectively.

The rest of the paper is organized as follows. In Sec. II we lay out the BLSSM contents that are necessary for SIDM calculation. In particular, we illustrate quantitatively why the bileptino SIDM is not viable. In Sec. III, we show the analytical derivation of sneutrino LSP from the DFP consideration. Furthermore, the parameter space of a sneutrino LSP is discussed in detail. In Sec. IV, we perform a numerical calculation of SIDM beyond the Born limit, where the solution of the Schrödinger equation at $r \rightarrow \infty$ captures the properties of elastic self-scattering. The relevant DM properties such as relic abundance involving the Sommerfeld enhancement (SE) effect and direct detection are also considered. We finally conclude in Sec. V.

\section{THE BLSSM DESCRIPTION}

In BLSSM, the chiral superfields are extended by a pair of bileptons $\left(\hat{\eta}_{1}, \hat{\eta}_{2}\right)$ and three generations of right-handed neutrinos $\hat{\nu}_{R_{i}}$. The neutrino mass generation and hierarchy can be obtained via the inverse-seesaw mechanism [49] or canonical type-I seesaw induced by SUSY breaking [50]. We ignore the neutrino mass issue in this paper and concentrate on SIDM in DPF SUSY. The complete particle contents and charge assignments are listed in Tables I and II. The relevant superpotential of BLSSM is given as

$$
\begin{aligned}
W= & Y_{u}^{i j} \hat{U}_{i} \hat{Q}_{j} \hat{H}_{u}-Y_{d}^{i j} \hat{D}_{i} \hat{Q}_{j} \hat{H}_{d}-Y_{e}^{i j} \hat{E}_{i} \hat{L}_{j} \hat{H}_{d}+\mu \hat{H}_{u} \hat{H}_{d} \\
& Y_{\eta}^{i j} \hat{\nu}_{R i} \hat{\eta}_{1} \hat{\nu}_{R j}+Y_{\nu}^{i j} \hat{L}_{i} \hat{H}_{u} \hat{\nu}_{R j}-\mu_{\eta} \hat{\eta}_{1} \hat{\eta}_{2},
\end{aligned}
$$

where $i, j$ denote family indices and all color and isospin indices are suppressed. The first line in Eq. (2) represents the conventional Yukawa interaction, as well as the $\mu$ term in the MSSM, while the second line stands for the additional interactions induced by the extended gauge group $U(1)_{B-L}$. The corresponding soft-breaking terms are given as

TABLE I. Vector superfields of the BLSSM and corresponding gauge couplings.

\begin{tabular}{lcccc}
\hline \hline Superfield & Spin $\frac{1}{2}$ & Spin 1 & Gauge group & Coupling \\
\hline$\hat{B}$ & $\lambda_{\tilde{B}}$ & $B$ & $U(1)_{Y}$ & $g_{1}$ \\
$\hat{W}$ & $\lambda_{\tilde{W}}$ & $W$ & $S U(2)_{L}$ & $g_{2}$ \\
$\hat{g}$ & $\lambda_{\tilde{g}}$ & $g$ & $S U(3)_{C}$ & $g_{3}$ \\
$\hat{B}^{\prime}$ & $\lambda_{\tilde{B}^{\prime}}$ & $B^{\prime}$ & $U(1)_{B-L}$ & $g_{B L}$ \\
\hline \hline
\end{tabular}


TABLE II. Chiral superfields of the BLSSM and their charges under the $U(1)_{Y} \otimes S U(2)_{L} \otimes S U(3)_{c} \otimes U(1)_{B-L}$ gauge group.

\begin{tabular}{lcc}
\hline \hline Superfield & $N_{G}$ & $U(1)_{Y} \otimes S U(2)_{L} \otimes S U(3)_{c} \otimes U(1)_{B-L}$ \\
\hline$\hat{Q}$ & 3 & $\frac{1}{6} \otimes \mathbf{2} \otimes \mathbf{3} \otimes \frac{1}{6}$ \\
$\hat{U}$ & 3 & $-\frac{2}{3} \otimes \mathbf{1} \otimes \overline{\mathbf{3}} \otimes-\frac{1}{6}$ \\
$\hat{D}$ & 3 & $\frac{1}{3} \otimes \mathbf{1} \otimes \mathbf{3} \otimes-\frac{1}{6}$ \\
$\hat{L}$ & 3 & $-\frac{1}{2} \otimes \mathbf{2} \otimes \mathbf{1} \otimes-\frac{1}{2}$ \\
$\hat{E}$ & 3 & $1 \otimes \mathbf{1} \otimes \mathbf{1} \otimes \frac{1}{2}$ \\
$\hat{\nu}_{R}$ & 3 & $0 \otimes \mathbf{1} \otimes \mathbf{1} \otimes \frac{1}{2}$ \\
$\hat{H}_{u}$ & 1 & $\frac{1}{2} \otimes \mathbf{2} \otimes \mathbf{1} \otimes 0$ \\
$\hat{H}_{d}$ & 1 & $-\frac{1}{2} \otimes \mathbf{2} \otimes \mathbf{1} \otimes 0$ \\
$\hat{\eta}_{1}$ & 1 & $0 \otimes \mathbf{1} \otimes \mathbf{1} \otimes-1$ \\
$\hat{\eta}_{2}$ & 1 & $0 \otimes \mathbf{1} \otimes \mathbf{1} \otimes 1$ \\
\hline \hline
\end{tabular}

$$
\begin{aligned}
\mathcal{L}_{\mathrm{BLSSM}}= & \mathcal{L}_{\mathrm{MSSM}}-M_{B B^{\prime}} \lambda_{\tilde{B}} \lambda_{\tilde{B}^{\prime}}-\frac{1}{2} M_{B^{\prime}} \lambda_{\tilde{B}^{\prime}} \lambda_{\tilde{B}^{\prime}} \\
& -m_{1}^{2}\left|\eta_{1}\right|^{2}-m_{2}^{2}\left|\eta_{2}\right|^{2}-m_{\nu, i j}^{2}\left(\tilde{\nu}_{R i}^{c}\right)^{*} \tilde{\nu}_{R j}^{c} \\
& -B_{\mu_{\eta}} \eta_{1} \eta_{2}+T_{\nu}^{i j} H_{u} \tilde{\nu}_{R i}^{c} \tilde{L}_{j}+T_{\eta}^{i j} \eta_{1} \tilde{\nu}_{R i}^{c} \tilde{\nu}_{R j}^{c} .
\end{aligned}
$$

We will see that $Y_{\eta}$ and $T_{\eta}=Y_{\eta} A_{\eta}$ play a crucial role in determining the SIDM cross section. Notice that $T_{\eta}$ leads to intrinsic mixing in the right-handed sneutrino sector, while $T_{\nu}$ leads to left-right mixing between the left-handed sneutrino sector and right-handed one. The conventional gravity mediation indicates that $T_{\nu}=Y_{\nu} A_{\nu}$; thus the leftright mixing can be neglected due to the vanishing $Y_{\nu}$. There is only right-right mixing left in this BLSSM, and mass splitting between the $C P$-even and $C P$-odd part yields

$$
\tilde{\nu}_{L}=\frac{1}{\sqrt{2}}\left(i \sigma_{L}+\phi_{L}\right), \quad \tilde{\nu}_{R}=\frac{1}{\sqrt{2}}\left(i \sigma_{R}+\phi_{R}\right),
$$

where $\left\{\phi_{L}, \phi_{R}\right\}$ are mixed into $C P$-even sneutrinos, and $\left\{\sigma_{L}, \sigma_{R}\right\}$ are mixed into $C P$-odd sneutrinos. Through Higgs states and bileptons receiving vacuum expectation values (VEVs), the electroweak and $U(1)_{B-L}$ symmetry are spontaneously broken into $U(1)_{e . m .}$. After symmetry breaking, the complex scalars are parametrized as

$$
\begin{aligned}
H_{d}^{0} & =\frac{1}{\sqrt{2}}\left(i \sigma_{d}+v_{d}+\phi_{d}\right), & H_{u}^{0} & =\frac{1}{\sqrt{2}}\left(i \sigma_{u}+v_{u}+\phi_{u}\right), \\
\eta_{1} & =\frac{1}{\sqrt{2}}\left(i \sigma_{1}+v_{1}+\phi_{1}\right), & \eta_{2} & =\frac{1}{\sqrt{2}}\left(i \sigma_{2}+v_{2}+\phi_{2}\right) .
\end{aligned}
$$

In analogy with $\tan \beta$ in the MSSM, we here denote the ratio of the two bilepton VEVs as $\tan \beta^{\prime}=v_{1} / v_{2}$. The $C P$ even Higgs sector is composed from the mixing of gauge eigenstates $\left\{\phi_{1}, \phi_{2}, \phi_{u}, \phi_{d}\right\}$. For a $C P$-odd Higgs, two of the four gauge eigenstates survive after gauge symmetry,

$$
m_{A^{0}}^{2}=\frac{2 B_{\mu}}{\sin 2 \beta}, \quad m_{A_{\eta}^{0}}^{2}=\frac{2 B_{\mu_{\eta}}}{\sin 2 \beta^{\prime}} .
$$

Since $B_{\mu}$ and $B_{\mu_{\eta}}$ are not related to DFP, they are relatively heavy so as not to be a candidate of the light force carrier. Meanwhile, the $C P$-even Higgs mass, especially $\phi_{1}$, is quantified as $m_{\eta_{1}}^{2}$, which is very tiny in DFP; thus it naturally leads to a light force carrier. For simplicity, we define $\eta_{1}$ as a lightest $C P$-even Higgs in order to recall that it comes mostly from the gauge eigenstate $\eta_{1}$.

A special property of BLSSM is that it gives rise to a gauge-kinetic mixing term with two Abelian gauge groups $U(1)_{B-L}$ and $U(1)_{Y}$ via

$$
\mathcal{L}=\frac{1}{4} \xi F_{\mu \nu}^{B-L} F^{Y, \mu \nu}
$$

where $\xi$ is a function of $\tilde{g}$ in Eq. (8). Even absent at tree level, it will be reintroduced via loop correction with the running effect of a renormalization group equation (RGE). In terms of a triangle form of the gauge coupling matrix [51], the bilepton contributions to the $Z$ mass vanish,

$$
\left(\begin{array}{ll}
g_{Y Y} & g_{Y B} \\
g_{B Y} & g_{B B}
\end{array}\right) \rightarrow\left(\begin{array}{cc}
g_{1} & \tilde{g} \\
0 & g_{B L}
\end{array}\right)
$$

and the gauge couplings are related by [52]

$$
\begin{aligned}
g_{1} & =\frac{g_{Y Y} g_{B B}-g_{Y B} g_{B Y}}{\sqrt{g_{B B}^{2}+g_{B Y}^{2}}}, \\
\tilde{g} & =\frac{g_{Y B} g_{B B}+g_{B Y} g_{Y Y}}{\sqrt{g_{B B}^{2}+g_{B Y}^{2}}}, \\
g_{B L} & =\sqrt{g_{B B}^{2}+g_{B Y}^{2}} .
\end{aligned}
$$

In addition, after electroweak and $U(1)_{B-L}$ breaking, the gauge-kinetic mixing further induces a mixing between the neutral SUSY particles from the MSSM and from the new sector, resulting in seven neutralinos in this model:

$$
\begin{aligned}
\chi_{1}^{0}= & c_{\tilde{B}} \tilde{B}+c_{\tilde{W}} \tilde{W}+c_{\tilde{H}_{u}} \tilde{H}_{u}+c_{\tilde{H}_{d}} \tilde{H}_{d} \\
& +c_{\tilde{\eta}_{1}} \tilde{\eta}_{1}+c_{\tilde{\eta}_{2}} \tilde{\eta}_{2}+c_{\tilde{B}^{\prime}} \tilde{B}^{\prime} .
\end{aligned}
$$

The LSP is then determined by the mixing coefficients $c_{i}$. For instance, in conventional MSSM with focus point SUSY, the Higgsino is the LSP with $c_{\tilde{\eta}_{1}} \sim c_{\tilde{\eta}_{2}} \sim 0.5$. DM in the BLSSM with a heavy $Z^{\prime}$ has been discussed in [43]. BLSSM with a light $Z^{\prime}$ is different and introduces a similar small hierarchy problem with MSSM from the perspective of a tadpole equation:

$$
m_{Z}^{2} \sim-2 m_{H_{u}}^{2}-2 \mu^{2}, \quad m_{Z^{\prime}}^{2} \sim-m_{\eta}^{2}-\mu_{\eta}^{2} .
$$

From the above equation, one can see that for heavy $Z^{\prime}$ around $\mathrm{TeV}$, there is no need to worry about the fine-tuning 
issue. However, a very light $Z^{\prime}$ is still allowed for tiny $g_{B L}$ [44], which forces us to consider the fine-tuning seriously. In order to retain naturalness, $m_{H_{u}}^{2}$ and $m_{\eta}^{2}$ should vanish at the electroweak scale simultaneously through the RGE running effect. Such a mechanism is called DFP SUSY,

$$
\begin{aligned}
{\left[\begin{array}{c}
m_{H_{u}}^{2}\left[Q_{\mathrm{GUT}}\right] \\
m_{q}^{2}\left[Q_{\mathrm{GUT}}\right] \\
m_{u}^{2}\left[Q_{\mathrm{GUT}}\right] \\
A_{t}^{2}\left[Q_{\mathrm{GUT}}\right]
\end{array}\right]=\left[\begin{array}{c}
m_{0}^{2} \\
m_{0}^{2}+\kappa_{0}^{\prime}-\frac{2 \kappa_{12}}{3} \\
m_{0}^{2}-\kappa_{0}^{\prime}-\frac{4 \kappa_{12}}{3} \\
6 \kappa_{12}
\end{array}\right] } \\
{\left[\begin{array}{c}
m_{H_{u}}^{2}\left[Q_{\mathrm{SUSY}}\right] \\
m_{q}^{2}\left[Q_{\mathrm{SUSY}}\right] \\
m_{u}^{2}\left[Q_{\mathrm{SUSY}}\right] \\
A_{t}^{2}\left[Q_{\mathrm{SUSY}}\right]
\end{array}\right]=\left[\begin{array}{c}
0 \\
\frac{m_{0}^{2}}{3}+\kappa_{0}^{\prime}-\frac{2 \kappa_{12}}{5} \\
\frac{2 m_{0}^{2}}{3}+\kappa_{0}^{\prime}-\frac{4 \kappa_{12}}{5} \\
\frac{2}{3} \kappa_{12}
\end{array}\right], }
\end{aligned}
$$

and

$$
\begin{aligned}
{\left[\begin{array}{c}
m_{\eta_{1}}^{2}\left[Q_{\mathrm{GUT}}\right] \\
m_{\nu_{R}}^{2}\left[Q_{\mathrm{GUT}}\right] \\
A_{\eta}^{2}\left[Q_{\mathrm{GUT}}\right.
\end{array}\right] } & =\left[\begin{array}{c}
m_{0}^{2} \\
\frac{43}{54} m_{0}^{2}-\frac{7}{20} \epsilon_{28} \\
7 \epsilon_{28}
\end{array}\right] \\
{\left[\begin{array}{c}
m_{\eta_{1}}^{2}\left[Q_{\mathrm{SUSY}}\right] \\
m_{\nu_{R}}^{2}\left[Q_{\mathrm{SUSY}}\right] \\
A_{\eta}^{2}\left[Q_{\mathrm{SUSY}}\right]
\end{array}\right] } & \rightarrow\left[\begin{array}{c}
0 \\
\frac{7}{54} m_{0}^{2}-\frac{7}{20} \epsilon_{28} \\
\frac{7}{100} \epsilon_{28}
\end{array}\right] .
\end{aligned}
$$

At first glance, since the $m_{\eta}^{2}$ becomes tiny through the DFP mechanism, the corresponding $\mu_{\eta}$ is small too. The bileptino $\left\{\tilde{\eta}_{1,2}\right\}$ thus becomes the LSP when $Z^{\prime}$ is much lighter than the $Z$ boson. We also mention that once the gauge kinetic term $\tilde{g}$ is tiny compared with $g_{B L}$, the $U(1)_{B-L}$ sector then decouples from the MSSM sector, which is the main point of obtaining SIDM in BLSSM. Furthermore, the hidden sector requirement in [24] is also the necessary condition of DFP SUSY.

It appears that the bileptino as the LSP with ultralight $Z^{\prime}$ as the mediator, i.e., $\left\{Z^{\prime}, \tilde{\eta}_{1}, \tilde{\eta}_{2}\right\}$ sector can furnish itself as SIDM without any fine-tuning issue. Nevertheless, it is not true when we consider the direct detection. As we know, the LSP is a Majorana type neutralino in MSSM which undergos spin-dependent scattering off nuclei via the exchange of the $Z$ boson. In the case of the bileptino in BLSSM, the interaction mediated by a light $Z^{\prime}$ is, however, a vectorlike type, which leads to a spin-independent interaction. Such an interaction can be constrained by the recent direct detection limits such as the LUX [53], XENON1T [54], and PandaX-II [55] experiments. The spin-independent scattering cross section can be written in terms of the spin-averaged squared matrix element,

$$
\sigma_{\mathrm{SI}}=\frac{\mu^{2}}{16 \pi m_{\chi}^{2} m_{A}^{2}}\left(\frac{1}{4} \sum_{s}|\mathcal{M}|^{2}\right)
$$

where $\mu$ is the reduced mass and $m_{A}$ is the mass of the target nucleus. The effective operator for spin-independent DM scattering through $Z^{\prime}$ exchange can be written as

$$
\mathcal{O}_{Z^{\prime}}=\lambda_{\chi Z^{\prime}} \lambda_{q Z^{\prime}} \frac{1}{m_{Z^{\prime}}}\left(\bar{\chi} \gamma^{\mu} \chi\right)\left(\bar{q} \gamma_{\mu} q\right)
$$

with $\lambda_{q Z^{\prime}}$ is the vertex between $Z^{\prime}$ and quarks, which is determined by the $U(1)_{B-L}$ gauge coupling $g_{B L}$. The coupling $\lambda_{\chi Z^{\prime}}$ is given explicitly as

$$
\lambda_{\chi Z^{\prime}}=g_{B L}\left(\left|N_{15}\right|^{2}-\left|N_{16}\right|^{2}\right)
$$

where $N_{15}$ and $N_{16}$ are the same as $c_{\eta_{1,2}}$ and stand for the bileptino fraction in LSP. Therefore, we find that the direct detection constraint can be evaded when the bileptino is composed with an equivalent fraction of $\tilde{\eta}_{1}, \tilde{\eta}_{2}$, i.e., $N_{15}=$ $N_{16}=0.5$ or very small $g_{\mathrm{BL}}$. The purity of the Dirac bileptino seems to escape from direct detections for almost canceled $\lambda_{\chi Z^{\prime}}$. However, under these circumstances it leads to a vanishing SIDM scattering cross section since $\lambda_{\chi Z^{\prime}}$ also determines the self-interacting scattering process. In turn, the large self-interacting cross section results in a large spin-independent cross section. This is the biggest challenge of BLSSM for the bileptino having the same coupling in direct detection and SIDM.

The conventional approach for escaping the direct detection constraint is to break the Dirac-type property of the bileptino. The degeneracy between bileptinos is broken by the nonvanishing expectation value of $\eta_{1}, \eta_{2}$. If the mass spitting is above $\mathrm{keV}$, which is the threshold of direct detection, i.e., the sensitivity of dark matter nucleus collision, there is only one Majorana bileptino left in the direct detection. The spin-independent scattering mediated by the $Z^{\prime}$ boson turns out to be a spin-dependent type whose constraint is relatively relaxed. Nevertheless, if the mass difference $\delta$ is as small as $\mathcal{O}(100) \mathrm{keV}$, the inelastic scattering process occurs and reintroduces the constraint from direct detection [56-58]. In BLSSM, the mass splitting ${ }^{1}$ between bileptinos is approximately proportional to $m_{Z^{\prime}}^{2} / 2 M_{B^{\prime}}$, which is below several hundred $\mathrm{keV}$ and thus constrained by inelastic scattering again. Finally, we conclude that the bileptino $\tilde{\eta}_{1,2}$ cannot be regarded as SIDM in BLSSM unless a very fine-tuned spectrum is imposed.

\footnotetext{
${ }^{1}$ The derivation of mass splitting could be found in the Appendix. The inelastic spin-independent constraints will be studied in an upcoming paper.
} 


\section{SNEUTRINO LSP CONSIDERATION}

The inconsistency between SIDM and BLSSM originates from the wrong illustration that DFP SUSY yields a Higgsino-like LSP, i.e., the bileptino in BLSSM. We point out that it is not necessarily the case even in the presence of a light $Z^{\prime}$. From Eq. (13), it is easy to find that $m_{\nu_{R}}^{2}$ can be as small as $m_{\eta_{1}}$ when large $A_{\eta}$ is given at the boundary. The sneutrino, especially the right-hand one, could also provide a plausible candidate of SIDM. In particular, the sneutrino mass can even vanish at one loop when $\epsilon_{28} \sim 10 / 27 m_{0}^{2}$.

After neglecting the kinetic mixing effect $\tilde{g}$ and setting $Y_{\nu}$ to be zero, we obtain the mass of right-handed sneutrinos in a compact form [41]:

$$
\begin{aligned}
m_{\tilde{\nu}^{+}}^{2}= & m_{\nu_{R}}^{2}+m_{Z^{\prime}}^{2}\left(\frac{1}{4} \cos 2 \beta^{\prime}+\frac{2 Y_{\eta}^{2}}{g_{B L}^{2}}\right) \\
& +m_{Z^{\prime}} \frac{\sqrt{2} Y_{\eta}}{g_{B L}}\left(A_{\eta} \sin \beta^{\prime}-\mu_{\eta} \cos \beta^{\prime}\right), \\
m_{\tilde{\nu}^{-}}^{2}= & m_{\nu_{R}}^{2}+m_{Z^{\prime}}^{2}\left(\frac{1}{4} \cos 2 \beta^{\prime}+\frac{2 Y_{\eta}^{2}}{g_{B L}^{2}}\right) \\
& -m_{Z^{\prime}} \frac{\sqrt{2} Y_{\eta}}{g_{B L}}\left(A_{\eta} \sin \beta^{\prime}-\mu_{\eta} \cos \beta^{\prime}\right) .
\end{aligned}
$$

Here the sign $+(-)$ stands for a $C P$-even $(C P$-odd $)$ sneutrino. In DFP SUSY, the $m_{0}$ and $A_{\eta}$ are related as $A_{\eta} \sim$ $\pm 0.18 m_{0}$ in order to guarantee $m_{\nu_{R}}$ to be the LSP. The typical value of $m_{0}$ in DFP is $(5-8) \times 10^{3} \mathrm{GeV}$ in order to increase the Higgs mass to $125 \mathrm{GeV}$. Large $m_{0}$, however, does not result in fine-tuning in the context of DFP SUSY. Thus, $A_{\eta}$ could be $\pm(900-1400) \mathrm{GeV}$ and could lead to a totally different result depending on the sign of $A_{\eta}$. The combination of $A_{\eta} \sin \beta^{\prime}-\mu \cos \beta^{\prime}$ further determines whether or not the $C P$-even sneutrino is the LSP. If $A_{\eta} \tan \beta^{\prime} \sim \mu_{\eta}$, we obtain a nearly degenerated right-hand sneutrino sector where the calculation of SIDM and SE must consider the inelastic scattering with an excited state [59-61]. However, a large $\tan \beta^{\prime} \sim 10$ is a necessary condition for DFP, which leads to a large $\mu_{\eta}$ in the degenerated sneutrino sector. That is unnatural so we ignore it in this paper. That is to say, the $\mu_{\eta}$ is suppressed not only by itself but also by small $\cot \beta^{\prime}$. The most important and relevant term for LSP determination is the sign of $A_{\eta}$.

In Fig. 1, we plot the behavior of the sneutrino as a function of the $Z^{\prime}$ mass after setting $m_{\tilde{\nu}_{R}}=100 \mathrm{GeV}$, $\tan \beta^{\prime}=10, Y_{\eta}=0.5, A_{\eta}= \pm 900 \mathrm{GeV}, \mu_{\eta}=100 \mathrm{GeV}$, and $g_{B L}=0.1$. The upper panel of Fig. 1 indicates that the $C P$-odd sneutrino is the LSP for positive $A_{\eta}$. This is because a positive $A_{\eta}$ drives the $C P$-odd senutrino lighter than $100 \mathrm{GeV}$. While a negative $A_{\eta}$ leads to a $C P$-even sneutrino LSP. We should mention that the $Z^{\prime}$ mass must
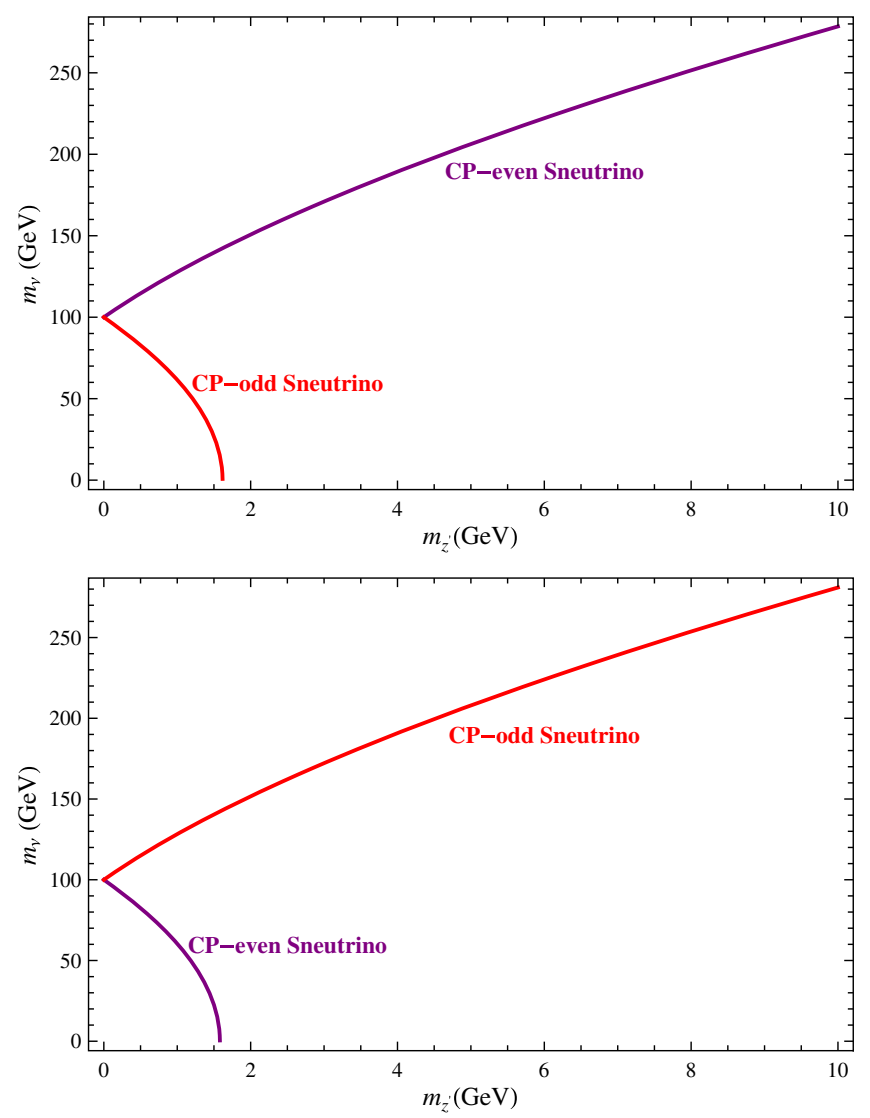

FIG. 1. The dependence of the sneutrino mass on $m_{Z^{\prime}}$ is given explicitly with $m_{\tilde{\nu}_{R}}=100 \mathrm{GeV}, \tan \beta^{\prime}=10, Y_{\eta}=0.5$, $A_{\eta}= \pm 900 \mathrm{GeV}, \mu_{\eta}=100 \mathrm{GeV}, g_{B L}=0.1$. The positive $A_{\eta}$ corresponds to a $C P$-odd sneutrino LSP, while negative $A_{\eta}$ corresponds to a $C P$-even sneutrino LSP.

be $2 \mathrm{GeV}$ in order to evade the dangerous tachyon problem of sneutrinos.

The only tree-level interactions responsible for dark matter annihilation are the Higgs mediator and $Z^{\prime}$ mediator. It is interesting to note that $Z^{\prime}$ must be removed since it couples to one $C P$-even and one $C P$-odd sneutrino at a time [43] that the coupling itself is always off-diagonal, i.e., $Z^{\prime} \tilde{\nu}_{1}^{+} \tilde{\nu}_{1}^{-}$. The inelastic scattering is equivalent to large $\mu_{\eta}$ in order to cancel $A_{\eta}$ contribution. Therefore, it ultimately leads to large fine-tuning. As a result, we only consider the Higgs mediator, which is now the light bilepton sector for a relatively heavy LSP sneutrino $\tilde{\nu}_{1}$. The effective Lagrangian of the hidden sector is then given as

$$
\begin{aligned}
\mathcal{L}_{\tilde{\nu}_{1} \tilde{\nu}_{1} \eta_{1}}= & \frac{1}{2} \partial^{\mu} \eta_{1} \partial_{\mu} \eta_{1}-\frac{m_{\eta}^{2}}{2} \eta_{1}^{2}+\frac{1}{2} \partial^{\mu} \tilde{\nu}_{1} \partial_{\mu} \tilde{\nu}_{1}-\frac{m_{\tilde{\nu}_{1}}}{2} \tilde{\nu}_{1}^{2} \\
& -\frac{\lambda}{2} \eta_{1} \tilde{\nu}_{1}^{2}-\frac{Y_{\eta}}{2} \eta_{1} \nu_{R}^{2}
\end{aligned}
$$

where $\lambda$ is the coupling constant between the two $\tilde{\nu}_{1}$ and $\eta_{1}$, which is used to mediate long-range interaction for SIDM 
and SE. The last term represents the interaction between the bilepton mediator and right-handed neutrinos, which comes from the superpotential (2). All the parameters in Eq. (18) are the function of SUSY breaking effects or superpotential couplings. Depending on whether the sneutrino LSP is $C P$ even or $C P$ odd, the coupling constant $\lambda$ is read as

$$
\begin{aligned}
\lambda_{\tilde{\nu}_{1} \tilde{\nu}_{1} \eta_{1}}^{+/-} & =\mp \sqrt{2} T_{\eta}-4 v_{\eta} Y_{\eta}^{2} \\
& =\mp \sqrt{2} A_{\eta} Y_{\eta}-4 \frac{m_{z^{\prime}}}{g_{B L}} \sin \beta^{\prime} Y_{\eta}^{2} .
\end{aligned}
$$

The Lagrangian in Eq. (18) describes the relevant interactions of relic density and the direct/indirect detection for sneutrino DM that have been discussed in [62]. The crucial difference is that in this paper we realize the SIDM in the sneutrino sector by retaining naturalness.

There is no severe direct detection constraint for righthanded sneutrino DM because the mediator bilepton has no tree level couplings to quarks. However, the direct detection constraint will be reintroduced if the bilepton mediator rapidly decays into SM final states before the BBN era [34,63-65], and bileptino DM seems to encounter the same obstacle. Such a dilemma can be solved easily in the framework of BLSSM where the mediator dominantly decays into right-handed neutrinos $\eta_{1} \rightarrow \nu_{R} \nu_{R}$ with the decay width,

$$
\Gamma\left[\eta_{1} \rightarrow \nu_{R} \nu_{R}\right]=Y_{\eta}^{2} \frac{\sqrt{m_{\eta_{1}}^{2} / 4-m_{\nu_{R}}^{2}}}{2 \pi} .
$$

Equation (20) there sets the lower bound of $Y_{\eta}$. The dominant channel for relic density and indirect detection is bilepton final states via $\tilde{\nu}_{1} \tilde{\nu}_{1} \rightarrow \eta_{1} \eta_{1}$ through t-channel and u-channel annihilation [66], plus the subsequent decay of bileptons.

At first sight, the total process is s-wave dominated and independent of the velocity of DM. However, it is not true when we consider the long-range Yukawa potential induced by the infinite exchange of bilepton mediators. It modifies the wave function of the annihilating DM pair at the origin, thus greatly altering the calculation of relic density and indirect detections. When only one partial wave is dominated in the annihilation process, the SE effect can be factorized as

$$
\sigma_{\mathrm{tot}}=S \sigma_{0}=|\psi(0)|^{2} \sigma_{0},
$$

where $S$ is the SE factor. The impact of the SE effect on relic abundance is encoded in the Boltzmann equation,

$$
\frac{d Y}{d x}=-\sqrt{\frac{\pi}{45}} \frac{g_{\star, \mathrm{eff}}^{1 / 2} M_{\mathrm{Pl}} m_{\tilde{\nu}_{1}}\left\langle\sigma v_{\text {rel }}\right\rangle}{x^{2}}\left(Y^{2}-Y_{\mathrm{eq}}^{2}\right),
$$

where $Y=n / s$, with $s=\left(2 \pi^{2} / 45\right) g_{\star s} T^{3}$ being the entropy density of the Universe. Moreover, $g_{\star, \text { eff }}$ and $g_{\star, s}$ are the energy and entropy degrees of freedom, respectively. The most important quantity for capturing particle physics input is the thermal average cross section,

$$
\begin{aligned}
\left\langle\sigma v_{\text {rel }}\right\rangle & =\int\left(\sigma v_{\text {rel }}\right)_{\text {tot }} f\left(v_{1}\right) f\left(v_{2}\right) d^{3} v_{1} d^{3} v_{2} \\
& =\int S_{0}\left(\sigma v_{\text {rel }}\right)_{0} f\left(v_{1}\right) f\left(v_{2}\right) d^{3} v_{1} d^{3} v_{2} .
\end{aligned}
$$

The factor $S_{0}$ encapsulates the effect of long-range interaction, which should be obtained by solving the radial part of the Schrödinger equation at the origin. Here we mention that the subscript 0 in $S_{0}$ corresponds to the s-wave contribution where only $l=0$ is considered though there were semianalytical studies for higher waves [67-69]. This is mainly because higher partial wave contributions are seriously suppressed by velocity.

Therefore, the total cross section is actually velocity dependent, which is consistent with the basic requirement of SIDM in order to be consistent with astrophysical observations. At low velocity, the cross section is enhanced to a large extent. Thus it puts strong constraints over the SIDM parameter space, which will be discussed in the next section. In particular, cosmic microwave background (CMB) reionization at ultralow velocity almost excludes the whole parameter space for s-wave annihilation SIDM [70]. This is the biggest constraint that we must consider in computing SIDM.

\section{SELF-INTERACTING DM CALCULATION}

The merit of SIDM is the relevant quantity $\sigma / m_{\tilde{\nu}_{1}}$, which undergoes velocity-dependent cross sections: for dwarf galaxies, $\sigma / m_{\tilde{\nu}_{1}} \sim 0.1-10 \mathrm{~cm}^{2} \mathrm{~g}^{-1}$ for velocity around $\mathcal{O}(10) \mathrm{km} \mathrm{s}^{-1}$, which is sufficient to solve the core-vscusp and too-big-to-fail problems, while the constraints from Milky Way sized galaxies and galaxy clusters require smaller $\sigma / m_{\tilde{\nu}_{1}}$, with $0.1-1 \mathrm{~cm}^{2} \mathrm{~g}^{-1}$ [71].

Since we are interested in the process with energy transfer in SIDM, the conventional total cross section $\sigma_{\text {tot }}=$ $\int d \Omega d \sigma / d \Omega$ is not suitable for estimating the process without energy transfer. Alternatively, we adapt two representative cross sections that have been discussed in plasma literature: one is the transfer cross section $\sigma_{T}$, and the other is the viscosity cross section $\sigma_{V}$,

$\sigma_{T}=\int d \Omega(1-\cos \theta) \frac{d \sigma}{d \Omega}, \quad \sigma_{V}=\int d \Omega \sin ^{2} \theta \frac{d \sigma}{d \Omega}$.

The transfer cross section $\sigma_{T}$ is used to regulate the forward scattering in terms of weight $(1-\cos \theta)$, which is usually used to study the Dirac fermion or complex scalar field. Meanwhile, the viscosity cross section $\sigma_{V}$ regulates forward and backward scattering simultaneously in terms of the weight $\sin ^{2} \theta$. Thus it is suitable for studying 
Majorana fermion or real scalar field DM. In our paper, the LSP is the real scalar particle $\tilde{\nu}_{1}$ and we use $\sigma_{V}$ throughout the paper.

The desirable cross section for SIDM is far larger than the typical WIMP freeze-out cross section, which strongly suggests the existence of a dark hidden sector $[72,73]$, i.e., a sneutrino LSP with bilepton mediator. We are now considering the interactions between two slowly moving $C P$-odd sneutrino DM mediated by a light force mediator $\eta$. Since the DM particles are nonrelativistic, the dominant process is the one exchanging multiple scalars $\eta_{1}$ while undergoing either an annihilation for relic abundance or scattering process for self-interaction. Such a process is well beyond the perturbation regime, i.e., the Born limit, and needs a resummation, which is equivalent to solving the Schrödinger equation in a reduced system. The induced Yukawa potential between the two particles alters the wave function of the reduced system at $r=0$, thus resulting in SE [67,74-77] for relic density and indirect detection. Meanwhile it also affects the wave function at $r \rightarrow \infty$, thus enhancing the self-interacting cross section. The underlying reason for the existence of enhancements on both $r \rightarrow 0$ and $r \rightarrow \infty$ comes from the similarity of the ladder diagrams between these two processes. The only difference comes from the fact that the annihilation diagram needs additional operator insertion for annihilating into SM particles, so the extent of these enhancements is not the same, though we can solve them simultaneously.

Here we define a dimensionless coupling constant which plays a crucial role in the nonperturbative regime as

$$
\alpha_{\eta}=\frac{1}{4 \pi}\left(\frac{\lambda}{2 m_{\tilde{\nu}_{1}}}\right)^{2}=\frac{Y_{\eta}^{2}}{4 \pi}\left(\frac{\sqrt{2} A_{\eta}-4 v_{\eta} Y_{\eta}}{2 m_{\tilde{\nu}_{1}}}\right)^{2},
$$

where $\tilde{\nu}_{1}$ is the mass eigenstate of the $C P$-odd sneutrino, which is related to the soft mass $m_{\tilde{\nu}_{R}}$ and $A_{\eta}$ through Eq. (17). In Fig. 2 we give a schematic overview of the dimensionless coupling constant $\alpha_{\eta}$ depending on $m_{\tilde{\nu}_{R}}$ and $A_{\eta}$ after setting $m_{Z^{\prime}}=1.5 \mathrm{GeV}, g_{B L}=0.01, \tan \beta^{\prime}=10$, and $Y_{\eta}=0.5$. It is easy to find in the vast parameter space that the magnitude of $\alpha$ is approximately $0.01-0.25$. The wave function with spherical symmetry can be expanded into spherical harmonics,

$$
\psi(r)=\sum_{l m} R_{l}(r) Y_{l m}(\theta, \phi) .
$$

The radial part of the wave function encodes all the relevant information that needs to be solved,

$$
\frac{1}{r^{2}} \frac{d}{d r}\left(r^{2} \frac{d R_{l}}{d r}\right)+\left(k^{2}-\frac{l(l+1)}{r^{2}}-2 \mu V(r)\right) R_{l}=0,
$$

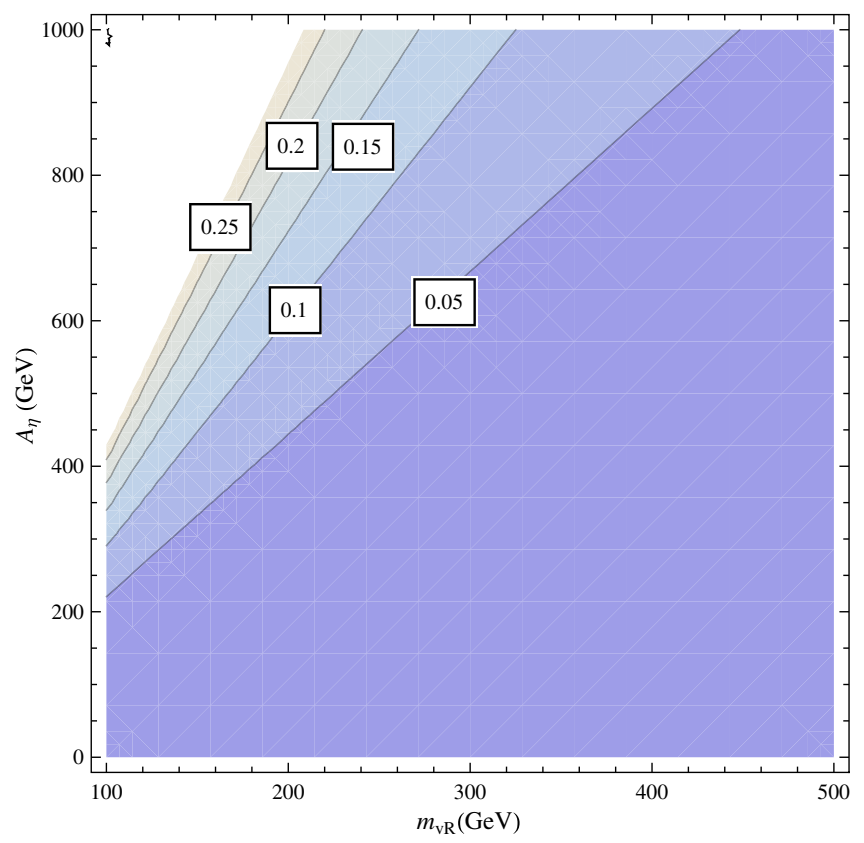

FIG. 2. The magnitude of $\alpha$ as a function of $m_{\tilde{\nu}_{R}}$ and $A_{\eta}$.

where $V(r)$ is the long-range potential that must be given at first. The Sommerfeld effect in MSSM is given in [78-80] explicitly through nonrelativistic effective field theory (NREFT). The derivation is quite similar with the quarkonium except that the sneutrino and bilepton are both scalars such that we do not need to worry about spin statistics. From the Lagrangian equation (18), the light mediator $\eta_{1}$ could be integrated out with the effective Lagrangian being given as [81]

$$
\begin{aligned}
S_{\text {eff }}= & \int d^{4} x\left(\frac{1}{2} \partial^{\mu} \tilde{\nu}_{1} \partial_{\mu} \tilde{\nu}_{1}+\frac{m_{\tilde{\nu}_{1}}^{2}}{2} \tilde{\nu}_{1}^{2}\right) \\
& +\frac{i}{2} \int d^{4} x d^{4} y j(x) D_{\eta_{1}}(x-y) j(y),
\end{aligned}
$$

where the current $j(x)$ is defined to be $\lambda \tilde{\nu}_{1}^{2} / 2$ and $D_{\eta_{1}}$ is the propagator of $\eta_{1}$. The residue field $\tilde{\nu}_{1}$ is further split up into nonrelativistic and relativistic parts in momentum space. The induced potential $V$ is equivalent to the interaction term with only nonrelativistic fields,

$$
\begin{aligned}
V & =\frac{i}{2} \int d^{4} x d^{4} y j(x) D_{\eta_{1}}(x-y) j(y) \\
& =i \frac{\lambda^{2}}{8} \int d^{4} x d^{4} y \tilde{\nu}_{1 \mathrm{NR}}^{2} D_{\eta_{1}}(x-y) \tilde{\nu}_{1 \mathrm{NR}}^{2}
\end{aligned}
$$

The leading order contribution of sneutrino annihilation into bileptons can be obtained from the imaginary part of box diagrams for self-scattering, i.e., the optical theorem. Therefore, the corresponding effective action is 


$$
\begin{aligned}
S_{\mathrm{ann}}= & -i \frac{A^{4}}{8} \int d^{4} x d^{4} y d^{4} z d^{4} w\left(\tilde{\nu}_{1 \mathrm{NR}} D_{\eta_{1}}(x-y) \tilde{\nu}_{1 \mathrm{NR}}\right. \\
& \left.D_{\tilde{\nu}_{1}}(y-z) \tilde{\nu}_{1 \mathrm{NR}} D_{\eta_{1}}(z-w) \tilde{\nu}_{1 \mathrm{NR}} D_{\tilde{\nu}_{1}}(w-z)\right) \\
& +(z \rightarrow w) .
\end{aligned}
$$

Here the two different terms are known to be the t-channel and u-channel box diagrams, respectively. The nonrelativistic form of the sneutrino is given as

$\tilde{\nu}_{1 \mathrm{NR}}=\frac{1}{\sqrt{2 m_{\tilde{\nu}_{1}}}}\left(\phi(x) \exp \left(-i m_{\tilde{\nu}_{1}} t\right)+\phi(x)^{\dagger} \exp \left(i m_{\tilde{\nu}_{1}} t\right)\right)$.

Combining Eqs. (29) and (30) with (31) yields the potential and annihilation cross section,

$$
\begin{aligned}
V & =-\frac{\lambda^{2}}{16 \pi m_{\tilde{\nu}_{1}}^{2}} \frac{e^{-m_{\eta_{1}} r}}{r}=-\frac{\alpha_{\eta}}{r} e^{-m_{\eta_{1}} r} \\
\left(\sigma v_{\mathrm{rel}}\right)_{0} & =\frac{3 \lambda^{4}}{512 \pi m_{\tilde{\nu}_{1}}^{6}} \frac{\left(1-m_{\eta_{1}}^{2} / m_{\tilde{\nu}_{1}}^{2}\right)^{1 / 2}}{\left[1-m_{\eta_{1}}^{2} / 4 m_{\tilde{\nu}_{1}}^{2}\right]^{2}} \\
& =\frac{3 \pi \alpha_{\eta}^{2}}{2 m_{\tilde{\nu}_{1}}^{2}} \frac{\left(1-m_{\eta_{1}}^{2} / m_{\tilde{\nu}_{1}}^{2}\right)^{1 / 2}}{\left[1-m_{\eta_{1}}^{2} / 4 m_{\tilde{\nu}_{1}}^{2}\right]^{2}}
\end{aligned}
$$

where in the first line of Eq. (32), we recognize the Yukawa potential. The negative sign means that the effective potential of scalar-scalar annihilation mediated by a light scalar particle is always attractive. The second line represents the annihilation cross section and the subscript 0 denotes that it does not consider loop and nonperturbative correction. It is also responsible for the finite lifetime of the bound state $[82,83]$ due to the decay of the reduced system where the bound state itself has an impact on dark matter freeze-out. Since our focus in this paper is to demonstrate the long-range effect of the bilepton $\eta_{1}$, we do not consider the bound state effects here and leave them for future work. A different, but completely equivalent approach [68,84-87] can reproduce the Yukawa potential. But the advantage of NREFT is that it not only calculates the potential but obtains the annihilation cross section. That is why we adapt this approach in this paper.

The Schrödinger equation with a Yukawa potential cannot be solved in an analytical form. The partial wave expansion method is thus introduced to cope with it numerically. The starting point is the assumption of the wave function,

$$
\begin{aligned}
& \psi(r) \sim e^{i k z}+f(\theta) \frac{e^{i k \cdot r}}{r}, \\
& f(\theta)=\sum_{l=0}^{\infty}(2 l+1) f_{l}(k) P_{l}(\cos \theta),
\end{aligned}
$$

where $P_{l}(\cos \theta)$ is the Bessel function, and $f_{l}(k)$ encodes the scattering information,

$$
f_{l}(k)=\frac{e^{2 i \delta_{l}(k)}-1}{2 i k} .
$$

The one that determines the scattering process is the phase shift $\delta_{l}$ of a certain partial wave. From $\delta_{l}$ we can obtain the differential cross section,

$$
\frac{d \sigma}{d \Omega}=\frac{1}{k^{2}}\left|(2 l+1) e^{i \delta_{l}} P_{l}(\cos \theta) \sin \delta_{l}\right|^{2},
$$

The integral viscosity cross section is expressed in terms of phase shifts $\delta_{l}$, via the analytical nonrelativistic formula,

$$
\sigma_{V}=\frac{4 \pi}{k^{2}} \sum_{l=0}^{\infty} \frac{(l+1)(l+2)}{(2 l+3)} \sin ^{2}\left(\delta_{l+2}-\delta_{l}\right) .
$$

In order to obtain $\sigma_{V}$, one must solve for $\delta_{l}$ from the asymptotic behavior of the radial function,

$$
\lim _{r \rightarrow \infty} R_{l}(r) \sim \cos \delta_{l} j_{l}(k r)-\sin \delta_{l} n_{l}(k r),
$$

where $j_{l}$ and $n_{l}$ are the spherical Bessel and Neumann functions, respectively. We explicitly calculate the viscosity cross section $\sigma_{V}$ from $\delta_{l}$ numerically. Equation (27) is not easy to implement in numerical calculation. We adapt the following parameter choice [35,84]:

$$
\begin{aligned}
\chi_{l} & =r R_{l}, & x & =\alpha_{\eta} m_{\tilde{\nu}_{1}} r, \\
a & =\frac{v}{2 \alpha_{\eta}}, & b & =\frac{\alpha_{\eta} m_{\tilde{\nu}_{1}}}{m_{\eta_{1}}} .
\end{aligned}
$$

In terms of Eq. (38), the radial wave function is reduced to be

$$
\left(\frac{d^{2}}{d x^{2}}+a^{2}-\frac{l(l+1)}{x^{2}}+\frac{1}{x} \exp (-x / b)\right) \chi_{l}=0 .
$$

Before showing our numerical results, we briefly discuss the effects of various constraints for SIDM on our model:

(i) Thermal relic abundance should yield $\Omega_{\tilde{\nu}_{1}} h^{2} \simeq 0.12$ and the impact of the SE on the leading order annihilation cross section in Eq. (32) should be taken into account. However, the naturalness argument requires the sneutrino mass to be no larger than $100 \mathrm{GeV}$ unless the bileptino becomes the LSP. Thus SE at freeze-out is negligible. We can use the relic density requirement to determine $\alpha_{\eta}$, with $m_{\tilde{\nu}_{1}}$, $m_{\eta_{1}}$ being input parameters.

(ii) Bilepton mediator decay provides a robust constraint on the sneutrino annihilation process from CMB observation and indirect detections. The enhancement at recombination time excludes most of the parameter space of DM annihilation dominated by 
the s-wave process. In BLSSM, the story is slightly different, given that the bilepton mediator is not directly decaying into SM final states but into righthanded neutrinos. Since the right-handed neutrino must be lighter than the mediator $\eta$ in order to allow (20) to be kinematically accessible, its subsequent decay process must be off shell into three fermions $[88,89]$. Such a decay width is so small due to the three-body phase space suppression that the righthanded neutrino could serve as a long-lived intermediator $[90,91]$. As the effective DM density that enters in the indirect signals is smeared by mediators, observations from indirect detection, especially gamma rays, are much less constraining than that of [70], where the vector mediator SIDM suffers from serious indirect detection constraints. Furthermore, the right-handed neutrino at the recombination scale does not insert energy for CMB; thus the retarded decay process also solves the $\mathrm{CMB}$ issue. One thus expects that there are no $\mathrm{CMB}$ and indirect detection constraints over SIDM parameter space. We will leave a detailed discussion of this issue to another work.

As a consequence, the only two important constraints in our model are relic abundance and the viscosity cross section by SIDM. In our model, the dominant DM annihilation channel is $\tilde{\nu}_{1} \tilde{\nu}_{1} \rightarrow \eta_{1} \eta_{1}$, and the corresponding annihilation cross section is given in Eq. (32). One can further eliminate $\alpha_{\eta}$ through the relic abundance constraint, leaving DM mass $m_{\tilde{\nu}_{1}}$ and mediator mass $m_{\eta_{1}}$ as two free parameters.

Here we follow Ref. [71] to investigate the effect of SIDM on different galaxy scales. For this purpose, we define $\left\langle\sigma_{V} / m_{\tilde{\nu}_{1}}\right\rangle_{v}$ as $\sigma_{V}$ averaged over a Maxwellian velocity distribution with the most probable velocity equal to $v\left(\mathrm{~km} \mathrm{~s}^{-1}\right)$, which corresponds to the typical velocity dispersion for a given galaxy. For dwarf galaxies, we choose $\left\langle\sigma_{V} / m_{\tilde{\nu}_{1}}\right\rangle_{v} \in 0.1-10 \mathrm{~cm}^{2} \mathrm{~g}^{-1}$ at $v=30 \mathrm{~km} \mathrm{~s}^{-1}$, while for galaxy clusters, there exist several upper limits: the constraint from the bullet cluster requires $\sigma_{V} / m_{\tilde{\nu}_{1}}<$ $1.25 \mathrm{~cm}^{2} \mathrm{~g}^{-1}$ at $68 \%$ confidential level (C.L.) [92] and the constraint from the ensemble of merging clusters imposes $\sigma_{V} / m_{\tilde{\nu}_{1}}<0.47 \mathrm{~cm}^{2} \mathrm{~g}^{-1}$ at $95 \%$ C.L. [93]. We thus choose more conservative regions such that $\left\langle\sigma_{V} / m_{\tilde{\nu}_{1}}\right\rangle_{v} \in 0.1-1.25 \mathrm{~cm}^{2} \mathrm{~g}^{-1}$ at $v=2000 \mathrm{~km} \mathrm{~s}^{-1}$ and $\left\langle\sigma_{V} / m_{\tilde{\nu}_{1}}\right\rangle_{v} \in 0.1-0.47 \mathrm{~cm}^{2} \mathrm{~g}^{-1}$ at $v=900 \mathrm{~km} \mathrm{~s}^{-1}$, respectively. Finally, for relic abundance, we require it to match the Planck observed value $\Omega_{\tilde{\nu}_{1}} h^{2}=0.1199 \pm 0.0027$ at 95\% C.L. [94]. Our results, shown in Fig. 3, illustrate the preferred parameter regions for different galaxy scales. The blue and green shaded bands show the regions preferred by dwarf galaxies, while the orange and purple regions present the parameter space preferred by galaxy clusters. Compared to dwarf galaxies, galaxy clusters hold smaller regions due to a more stringent requirement on $\sigma_{V} / m_{\tilde{\nu}_{1}}$, and

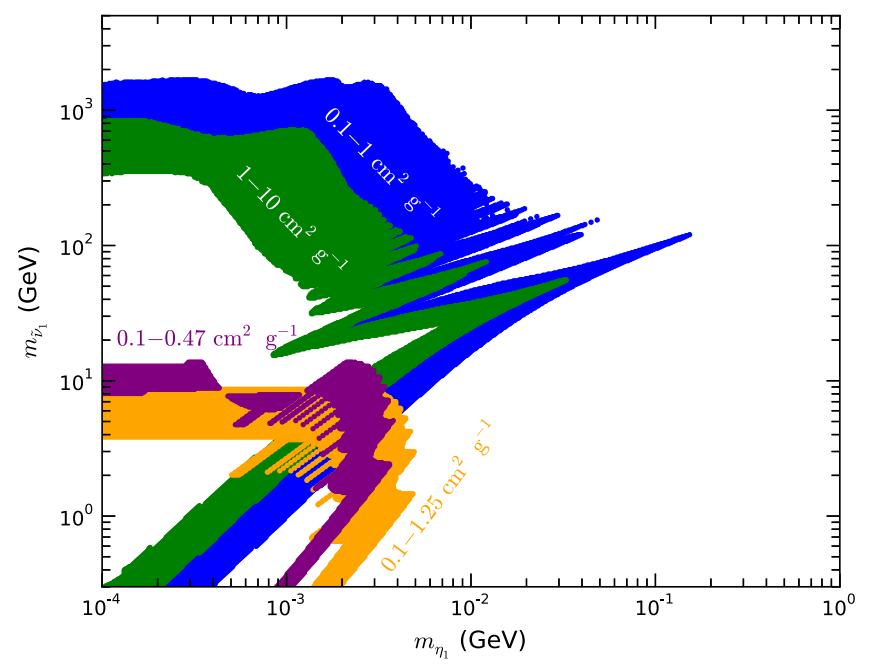

FIG. 3. Preferred parameter regions by relic abundance and SIDM in the $\left[m_{\eta_{1}}, m_{\tilde{\nu}_{1}}\right]$ plane. The blue and green shaded regions correspond to $\left\langle\sigma_{V} / m_{\tilde{\nu}_{1}}\right\rangle_{30} \in 0.1-10 \mathrm{~cm}^{2} \mathrm{~g}^{-1}$, which would significantly affect astrophysical observables at the scale of dwarf galaxies. Also shown are regions preferred by galaxy clusters with $\left\langle\sigma_{V} / m_{\tilde{\nu}_{1}}\right\rangle_{2000} \in 0.1-1.25 \mathrm{~cm}^{2} \mathrm{~g}^{-1}$ (orange shaded) and $\left\langle\sigma_{V} / m_{\tilde{\nu}_{1}}\right\rangle_{900} \in 0.1-0.47 \mathrm{~cm}^{2} \mathrm{~g}^{-1}$ (purple shaded).

the common parameter space can exist for $m_{\tilde{\nu}_{1}} \sim 1-10 \mathrm{GeV}$ and $m_{\eta_{1}} \sim 1-4 \mathrm{MeV}$. Moreover, for coupling $\alpha_{\eta}$, one observes from Eq. (32) that the annihilation cross section is insensitive to the mediator mass for $m_{\eta_{1}} \ll m_{\tilde{\nu}_{1}} . \alpha_{\eta}$ thus increases monotonically with DM mass $m_{\tilde{\nu}_{1}}$ and varies from $5 \times 10^{-6}$ to $3 \times 10^{-2}$ (from $5 \times 10^{-6}$ to $3 \times 10^{-4}$ ) for dwarf galaxies (galaxy clusters) within our interested parameter regions.

\section{CONCLUSION}

In this paper, we have discussed in detail the realization of SIDM within the framework of the $U(1)_{B-L}$ extension of MSSM and the DFP mechanism. The right-handed sneutrino serves as the LSP in most of the parameter space for a nonvanishing soft trilinear coupling $T_{\eta}$. It is thus compatible with the requirement of DFP SUSY without introducing dangerous direct detection limits. Its relic abundance is achieved via a sneutrino annihilating into a pair of bileptons, which also serve as a light mediator in DM selfscattering. More interestingly, the annoying CMB constraint can be escaped by the retarded decay of right-handed neutrinos. The numerical calculation indicates that SIDM can be realized in our model from the scales of a dwarf galaxy to a galaxy cluster.

\section{ACKNOWLEDGMENTS}

We would like to thank Wenyu Wang for help on numerical calculation, and Fei Wang for helpful discussion. B. Z. is supported by the National Science Foundation of 
China (Grants No. 11747026 and No. 11575151). Y. L. is also supported by the Natural Science Foundation of Shandong Province (Grant No. ZR2016JL001).

\section{APPENDIX: DERIVATION OF MASS SPLITTING IN BILEPTINOS}

Here we give a derivation of the mass splitting for bileptinos in BLSSM. The procedure is similar to that in $[95,96]$. In BLSSM, the mass term for bileptinos $\tilde{\eta}_{1,2}$ is given explicitly from the superpotential,

$$
\mathcal{L}_{\text {bileptino }}=-\mu_{\eta} \tilde{\eta}_{1} \tilde{\eta}_{2}+\text { H.c. }
$$

$\tilde{\eta}_{1,2}$ is the Dirac fermion if no mixing effect is introduced. As a result, an accidental global $U(1)$ symmetry is induced where $\tilde{\eta}_{1,2}$ could be rotated with each other. The mixing effect which divides the Dirac fermion into two nearly degenerated Majorana fermion thus must come from the $U(1)$ breaking operator. Considering the dimension-five operator,

$$
\mathcal{L}_{\text {eff }}=\sum_{i=1}^{2} c_{i} \mathcal{O}_{i}+\text { H.c. }
$$

where

$$
\begin{aligned}
& \mathcal{O}_{1}=\eta_{2} \tilde{\eta}_{1} \eta_{2} \tilde{\eta}_{1}, \\
& \mathcal{O}_{2}=\eta_{1} \tilde{\eta}_{2} \eta_{1} \tilde{\eta}_{2} .
\end{aligned}
$$

These two operators can be obtained through integrating out the corresponding heavy blino, which is the superpartner of the $U(1)_{B-L}$ gauge boson. The Wilson coefficients give rise to mass splitting for bileptinos that can be found in the mass matrix directly,

$$
\mathcal{L}=-\frac{1}{2}\left(\tilde{\eta}_{1}, \tilde{\eta}_{2}\right) \mathcal{M}\left(\begin{array}{l}
\tilde{\eta}_{1} \\
\tilde{\eta}_{2}
\end{array}\right)
$$

with

$$
\mathcal{M}=\left(\begin{array}{cc}
-\cos ^{2} \beta^{\prime} c_{1} v_{\eta}^{2} & -\mu_{\eta} \\
-\mu_{\eta} & -\sin ^{2} \beta^{\prime} c_{2} v_{\eta}^{2}
\end{array}\right) .
$$

The symmetric mass matrix $\mathcal{M}$ can be diagonalized, and the resulting mass eigenvalues are

$$
\begin{aligned}
& m_{1}=\mu-\frac{1}{2}\left(c_{1} \cos ^{2} \beta^{\prime}+c_{2} \sin ^{2} \beta^{\prime}\right) v_{\eta}^{2}, \\
& m_{2}=\mu+\frac{1}{2}\left(c_{1} \cos ^{2} \beta^{\prime}+c_{2} \sin ^{2} \beta^{\prime}\right) v_{\eta}^{2} .
\end{aligned}
$$

Therefore, the mass splitting is found to be

$$
\delta=m_{2}-m_{1}=\left(c_{1} \cos \beta^{\prime}+c_{2} \sin \beta^{\prime}\right) v_{\eta}^{2} .
$$

The Wilson coefficients $c_{1}$ and $c_{2}$ can be obtained by matching the blino-bileptino-bilepton vertex,

$$
c_{1}=c_{2}=\frac{g_{\mathrm{BL}}^{2}}{4 M_{B^{\prime}}^{2}} .
$$

The resulting mass splitting is

$$
\delta=\frac{m_{Z^{\prime}}^{2}}{2 M_{B^{\prime}}} .
$$

[1] S. P. Martin, Adv. Ser. Dir. High Energy Phys. 18, 1 (1998).

[2] S. R. Coleman and J. Mandula, Phys. Rev. 159, 1251 (1967).

[3] H. P. Nilles, Phys. Rep. 110, 1 (1984).

[4] G. Bertone, D. Hooper, and J. Silk, Phys. Rep. 405, 279 (2005).

[5] G. Aad et al. (ATLAS Collaboration), Phys. Lett. B 716, 1 (2012).

[6] S. Chatrchyan et al. (CMS Collaboration), Phys. Lett. B 716, 30 (2012).

[7] U. Ellwanger, M. R. de Traubenberg, and C. A. Savoy, Nucl. Phys. B492, 21 (1997).

[8] U. Ellwanger and C. Hugonie, Mod. Phys. Lett. A 22, 1581 (2007).

[9] U. Ellwanger, C. Hugonie, and A. M. Teixeira, Phys. Rep. 496, 1 (2010).
[10] J. L. Feng, K. T. Matchev, and T. Moroi, Phys. Rev. D 61, 075005 (2000).

[11] J. L. Feng, Annu. Rev. Nucl. Part. Sci. 63, 351 (2013).

[12] J. L. Feng, K. T. Matchev, and D. Sanford, Phys. Rev. D 85, 075007 (2012).

[13] J. L. Feng and D. Sanford, Phys. Rev. D 86, 055015 (2012).

[14] J. L. Feng and K. T. Matchev, Phys. Rev. D 63, 095003 (2001).

[15] D. Horton and G. G. Ross, Nucl. Phys. B830, 221 (2010).

[16] F. Brummer and W. Buchmuller, J. High Energy Phys. 05 (2012) 006.

[17] T. T. Yanagida and N. Yokozaki, Phys. Lett. B 722, 355 (2013).

[18] K. Agashe, Phys. Rev. D 61, 115006 (2000).

[19] P. Draper, J. L. Feng, P. Kant, S. Profumo, and D. Sanford, Phys. Rev. D 88, 015025 (2013). 
[20] R. Ding, T. Li, F. Staub, and B. Zhu, J. High Energy Phys. 03 (2014) 130.

[21] C. S. Un and O. Ozdal, Phys. Rev. D 93, 055024 (2016).

[22] L. Delle Rose, S. Khalil, S. J. D. King, C. Marzo, S. Moretti, and C. S. Un, Phys. Rev. D 96, 055004 (2017).

[23] L. Delle Rose, S. Khalil, S. J. D. King, C. Marzo, S. Moretti, and C. S. Un, Proc. Sci., DIS2017 (2018) 301 [arXiv: 1706.01301].

[24] B. Zhu, F. Staub, and R. Ding, Phys. Rev. D 96, 035038 (2017).

[25] S.-M. Zhao, T.-F. Feng, Z.-J. Yang, H.-B. Zhang, X.-X. Dong, and T. Guo, Eur. Phys. J. C 77, 102 (2017).

[26] N. Arkani-Hamed, A. Delgado, and G. F. Giudice, Nucl. Phys. B741, 108 (2006).

[27] R. A. Flores and J. R. Primack, Astrophys. J. 427, L1 (1994).

[28] M. Boylan-Kolchin, J. S. Bullock, and M. Kaplinghat, Mon. Not. R. Astron. Soc. 415, L40 (2011).

[29] M. Boylan-Kolchin, J. S. Bullock, and M. Kaplinghat, Mon. Not. R. Astron. Soc. 422, 1203 (2012).

[30] S.-H. Oh, W. J. G. de Blok, E. Brinks, F. Walter, and R. C. Kennicutt, Jr., Astron. J. 141, 193 (2011).

[31] M. G. Walker, arXiv:1205.0311.

[32] A. H. G. Peter, M. Rocha, J. S. Bullock, and M. Kaplinghat, Mon. Not. R. Astron. Soc. 430, 105 (2013).

[33] S. Tulin, H.-B. Yu, and K. M. Zurek, Phys. Rev. Lett. 110, 111301 (2013).

[34] M. Kaplinghat, S. Tulin, and H.-B. Yu, Phys. Rev. D 89, 035009 (2014).

[35] S. Tulin, H.-B. Yu, and K. M. Zurek, Phys. Rev. D 87, 115007 (2013).

[36] P. Ko and Y. Tang, J. Cosmol. Astropart. Phys. 05 (2014) 047.

[37] F. Wang, W. Wang, J. M. Yang, and S. Zhou, Phys. Rev. D 90, 035028 (2014).

[38] H.-J. Kang and W. Wang, Commun. Theor. Phys. 65, 499 (2016).

[39] S. Tulin and H.-B. Yu, Phys. Rep. 730, 1 (2018).

[40] G. G. Ross, K. Schmidt-Hoberg, and F. Staub, J. High Energy Phys. 08 (2012) 074.

[41] B. O'Leary, W. Porod, and F. Staub, J. High Energy Phys. 05 (2012) 042.

[42] M. Hirsch, M. Malinsky, W. Porod, L. Reichert, and F. Staub, J. High Energy Phys. 02 (2012) 084.

[43] L. Basso, B. O'Leary, W. Porod, and F. Staub, J. High Energy Phys. 09 (2012) 054.

[44] Yu. Ya. Komachenko and M. Yu. Khlopov, Yad. Fiz. 51, 1081 (1990) [Sov. J. Nucl. Phys. 51, 692 (1990)].

[45] J. L. Feng, B. Fornal, I. Galon, S. Gardner, J. Smolinsky, T. M. P. Tait, and P. Tanedo, Phys. Rev. Lett. 117, 071803 (2016).

[46] J. L. Feng, B. Fornal, I. Galon, S. Gardner, J. Smolinsky, T. M. P. Tait, and P. Tanedo, Phys. Rev. D 95, 035017 (2017).

[47] P.-H. Gu and X.-G. He, Nucl. Phys. B919, 209 (2017).

[48] O. Seto and T. Shimomura, Phys. Rev. D 95, 095032 (2017).

[49] F. Bazzocchi, D. G. Cerdeno, C. Munoz, and J. W. F. Valle, Phys. Rev. D 81, 051701 (2010).

[50] N. Arkani-Hamed, L. J. Hall, H. Murayama, D. TuckerSmith, and N. Weiner, Phys. Rev. D 64, 115011 (2001).
[51] R. M. Fonseca, M. Malinsky, W. Porod, and F. Staub, Nucl. Phys. B854, 28 (2012).

[52] P. H. Chankowski, S. Pokorski, and J. Wagner, Eur. Phys. J. C 47, 187 (2006).

[53] D. S. Akerib et al. (LUX Collaboration), Phys. Rev. Lett. 118, 021303 (2017).

[54] E. Aprile et al. (XENON Collaboration), arXiv:1805.12562.

[55] X. Cui et al. (PandaX-II Collaboration), Phys. Rev. Lett. 119, 181302 (2017).

[56] D. Tucker-Smith and N. Weiner, Phys. Rev. D 64, 043502 (2001).

[57] D. Tucker-Smith and N. Weiner, Phys. Rev. D 72, 063509 (2005).

[58] S. Chang, G. D. Kribs, D. Tucker-Smith, and N. Weiner, Phys. Rev. D 79, 043513 (2009).

[59] T. R. Slatyer, J. Cosmol. Astropart. Phys. 02 (2010) 028.

[60] A. Das and B. Dasgupta, Phys. Rev. Lett. 118, 251101 (2017).

[61] Y. Zhang, Phys. Dark Universe 15, 82 (2017).

[62] L. Delle Rose, S. Khalil, S. J. D. King, S. Kulkarni, C. Marzo, S. Moretti, and C. S. Un, arXiv:1712.05232.

[63] E. Del Nobile, M. Kaplinghat, and H.-B. Yu, J. Cosmol. Astropart. Phys. 10 (2015) 055.

[64] N. Bernal, X. Chu, C. Garcia-Cely, T. Hambye, and B. Zaldivar, J. Cosmol. Astropart. Phys. 03 (2016) 018.

[65] K. Kainulainen, K. Tuominen, and V. Vaskonen, Phys. Rev. D 93, 015016 (2016); 95, 079901(E) (2017).

[66] M. Pospelov, A. Ritz, and M. B. Voloshin, Phys. Lett. B 662, 53 (2008).

[67] S. Cassel, J. Phys. G 37, 105009 (2010).

[68] R. Iengo, J. High Energy Phys. 05 (2009) 024.

[69] S. El Hedri, A. Kaminska, and M. de Vries, Eur. Phys. J. C 77, 622 (2017).

[70] T. Bringmann, F. Kahlhoefer, K. Schmidt-Hoberg, and P. Walia, Phys. Rev. Lett. 118, 141802 (2017).

[71] M. Kaplinghat, S. Tulin, and H.-B. Yu, Phys. Rev. Lett. 116, 041302 (2016).

[72] M. Pospelov and A. Ritz, Phys. Lett. B 671, 391 (2009).

[73] K. K. Boddy, J. L. Feng, M. Kaplinghat, and T. M. P. Tait, Phys. Rev. D 89, 115017 (2014).

[74] J. Hisano, S. Matsumoto, M. Nagai, O. Saito, and M. Senami, Phys. Lett. B 646, 34 (2007).

[75] M. Cirelli, A. Strumia, and M. Tamburini, Nucl. Phys. B787, 152 (2007).

[76] J. L. Feng, M. Kaplinghat, and H.-B. Yu, Phys. Rev. D 82, 083525 (2010).

[77] J. B. Dent, S. Dutta, and R. J. Scherrer, Phys. Lett. B 687, 275 (2010).

[78] M. Beneke, C. Hellmann, and P. Ruiz-Femenia, J. High Energy Phys. 03 (2013) 148; 10 (2013) 224(E).

[79] C. Hellmann and P. Ruiz-Femenía, J. High Energy Phys. 08 (2013) 084.

[80] M. Beneke, C. Hellmann, and P. Ruiz-Femenia, J. High Energy Phys. 05 (2015) 115.

[81] S. Aarssen, Ph.D. thesis, Universität Hamburg, 2013.

[82] K. Petraki, M. Postma, and M. Wiechers, J. High Energy Phys. 06 (2015) 128.

[83] K. Petraki, M. Postma, and J. de Vries, J. High Energy Phys. 04 (2017) 077. 
[84] M. R. Buckley and P. J. Fox, Phys. Rev. D 81, 083522 (2010).

[85] A. Hryczuk, R. Iengo, and P. Ullio, J. High Energy Phys. 03 (2011) 069.

[86] A. Hryczuk, Phys. Lett. B 699, 271 (2011).

[87] Z.-P. Liu, Y.-L. Wu, and Y.-F. Zhou, Phys. Rev. D 88, 096008 (2013).

[88] M. C. Gonzalez-Garcia, A. Santamaria, and J. W. F. Valle, Nucl. Phys. B342, 108 (1990).

[89] M. Escudero, N. Rius, and V. Sanz, J. High Energy Phys. 02 (2017) 045.

[90] D. Kim, J.-C. Park, and S. Shin, J. High Energy Phys. 04 (2018) 093.
[91] X. Chu, S. Kulkarni, and P. Salati, J. Cosmol. Astropart. Phys. 11 (2017) 023.

[92] S. W. Randall, M. Markevitch, D. Clowe, A. H. Gonzalez, and M. Bradac, Astrophys. J. 679, 1173 (2008).

[93] D. Harvey, R. Massey, T. Kitching, A. Taylor, and E. Tittley, Science 347, 1462 (2015).

[94] P. A. R. Ade et al. (Planck Collaboration), Astron. Astrophys. 594, A13 (2016).

[95] N. Nagata and S. Shirai, J. High Energy Phys. 01 (2015) 029.

[96] R. Krall and M. Reece, Chin. Phys. C 42, 043105 (2018). 